\title{
Short Term Electricity Load Forecasting using Smart Grid AI-Prediction Simulator (SGAIPS)
}

\author{
Fouad Mountassir \\ National School of Applied Sciences of EL Jadida \\ LTI Laboratory (Information Technology Laboratory) \\ El-Jadida, Morocco
}

\author{
Mohammed Bousmah \\ National School of Applied Sciences of EL Jadida \\ LTI Laboratory (Information Technology Laboratory) \\ El-Jadida, Morocco
}

\begin{abstract}
Electricity Demand Forecasting techniques with Artificial Intelligence based on Machine Learning and Deep Learning Models have been widely used in the past 20 years. These techniques play an important role in the construction of next generation of Smart Grid. However, applied machine learning in this real world presents challenges, it requires resources (Datasets from Smart Meters and other IoT-connected devices), skills (Machine Learning Algorithms, python or other programming languages) and knowledge (conventional load forecasting methods, intelligent load forecasting methods). For this purpose, we have developed, a new Smart Grid Artificial Intelligence Prediction Simulator called SGAIPS, that can provide a systematic short term electricity load forecasting based on Smart meters and Machine Learning algorithms. The starting point for using SGAIPS is the construction of dataset by loading or generating smart meters' data. The next step is to create, train and test the machine learning models. And the final step is to predict the energy consumption. SGAIPS is a zero code Simulator for Smart Grid, that aims to simplify the creation and implementation of virtual smart meters, machine learning models and intelligent forecasting methods.
\end{abstract}

\section{Keywords}

Short-Term Load Forecasting; Artificial Intelligence; Smart Meters; Smart Grid; Prediction Simulator.

\section{INTRODUCTION}

Traditional power networks are being transformed into smart grids to solve the problems of existing electric system due to the flow of unidirectional information, wasted energy, and growing demand for energy, reliability and security [1].

With the Smart Grid technology, we can also:

- Increase integration of large-scale renewable energy systems,

- Better integration of customer-owner power generation systems, including renewable energy systems.

As shown in the Figure 1, Smart Grid integrates computing and two-way communication capabilities with existing power infrastructures. They cover all levels of the energy value chain and are not limited to smart meters, they make intelligent use of sensors, embedded computing and digital communications to make the electricity network observable (measurement and visualization capacity), controllable (manipulation and optimization capacity), automated (ability to adapt and self-correcting) and fully integrated (full interoperability with existing systems and ability to incorporate a diverse range of energy sources) [1].

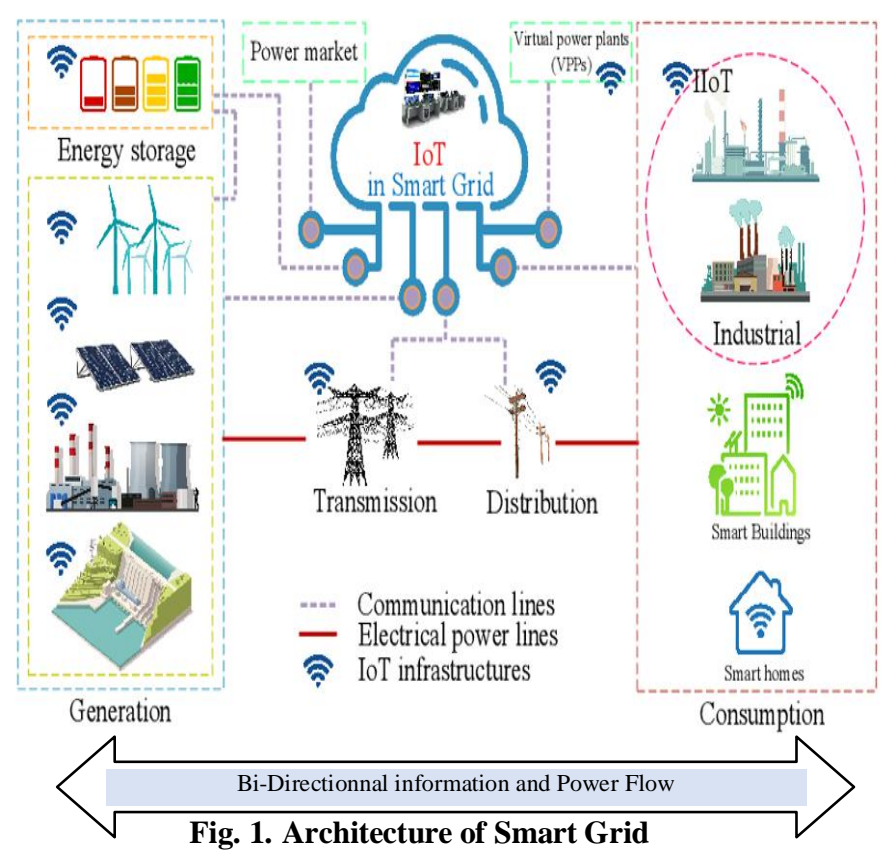

Electrical Load Forecasting (ELF) plays an important role in the construction of smart grid system. Indeed, with these methods, we can: (1) avoid unexpected power outages, (2) reduce operating costs, (3) enable efficiency and stability (4) avoid increased demand in electrical energy [2].

ELF has become a priority for different types of stakeholders to make the right decisions about future energy development at regional, national and global levels. However, there is no precise standard for classifying the ELF approaches. The criterion for choosing a forecasting approach is based on several elements, including the forecast horizon, the forecasting methods, the relevance and availability of the historical data, etc. [3]

Therefore, choosing the right approach to forecast the load depends primarily on the time horizon of the forecast and the load forecasting methods.

In terms of time horizon of the forecast, some authors have divided ELF into three categories [4, 5, 6, 7]: short-term forecasts, medium-term forecasts, and long-term forecasts. Other researchers go for classifying load forecasting into four groups $[8,9,10,11]$ : long-term forecasts, mid-term forecasts, short-term forecasts, and very short-term forecasts, as follows

- Long-term load forecasting (LTLF): is for more than one year to 20 years ahead. This type of forecast is fundamental for strategic planning, construction of new generations, and develops the power supply and delivery system (generation units, transmission system, and distribution system). 
- Medium-term load forecasting (MTLF): is usually for a week up to a year, which is used for maintenance scheduling and planning fuel purchases as well as energy trading and revenue assessment for the utilities.

- Short-term load forecasting (STLF): is for intervals ranging from one hour to a week, it is very important for day-to-day operations of a utility, schedule the generation and transmission of electricity.

- Ultra/very short-term load forecasting (VSTLF): ranges from a few minutes to an hour ahead and is used for real-time control.

In terms of load forecasting methods, it could be mainly categorized into two types: Conventional Load Forecasting Methods (CLFM) and Intelligent Load Forecasting Methods (ILFM) [12].

- In Conventional Load Forecasting Methods (CLFM), the model of the load is built upon the relationship between load and load-impacting factors, the adequacy of the model being assessed based on the forecast errors as model residuals. ARMA, ARIM, ARMAX or ARIMAX are widely used time series methods $[13,14,15]$.

- In Intelligent Load Forecasting Methods (ILFM), Machine Learning, Deep Learning and AI techniques constitute the founding blocks for this category. It mostly includes Support Vector Machine (SVM) [16], Artificial Neural Network (ANN) [17], Recurrent Neural Network (RNN) and Long Short-Term Memory (LSTM) [18, 19] and other AI blocks.

Machine Learning is a branch of Artificial Intelligence (AI) that focuses on using data and algorithms to solve problems without specific computer programming [20]. As shown in Figure 2, ML is a multi-disciplinary field having a wide-range of research domains reinforcing its existence [21].

Deep learning as illustrated in Figure 3, is part of a broader family of Machine Learning methods based on artificial neural networks with representation learning [22, 23]. Learning can be supervised, unsupervised or reinforced [24]. It has expanded significantly over the past few years, especially since 2013 thanks to the availability of data and GPUs that can perform parallel processing faster.

Although a large number of Intelligent Load Forecasting Methods (ILFM) have been proposed to improve prediction results, little research has been conducted to facilitate their deployment.

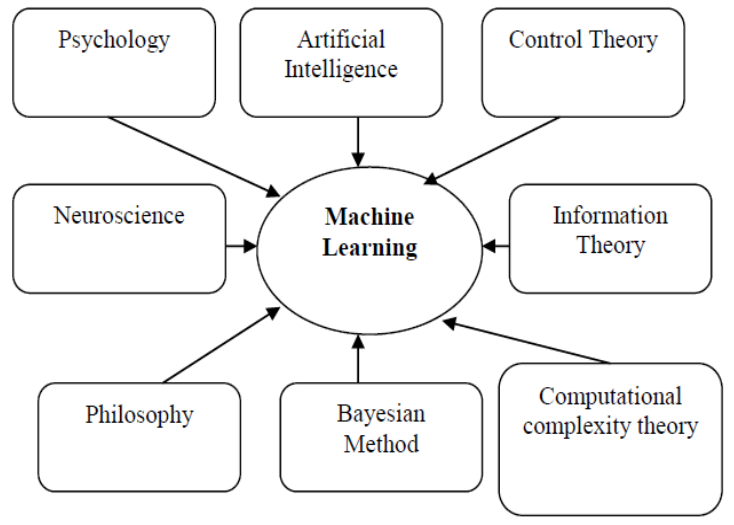

Fig. 2. The Multi-disciplinary ML

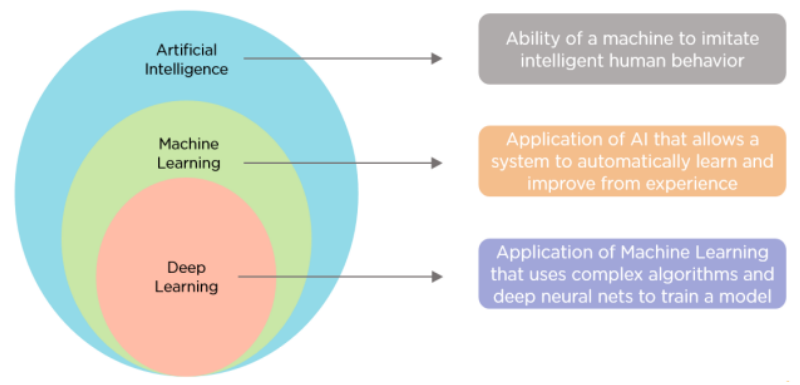

Fig. 3. AI vs Machine Learning vs Deep Learning.

For this reason, we are interested in answering the following research question:

RQ: Can we simplify the deployment of Intelligent Load Forecasting Methods in Smart Grids?

The objective of this article is to answer this question and build a new Smart Grid Artificial Intelligence Prediction Simulator called SGAIPS, delivered as SaaS (software-as-a-Service), that aims to simplify the deployment of Intelligent Load Forecasting Methods (ILFM) in smart grids. We focus on the problem of short-term load forecasting using ILFM and their deployment.

The rest of this article is organized as follows: In the next section, we present the related works about this research question. Section III presents our proposed model used in this work. In Section IV, the SGAIPS Architecture and deployment are discussed. Finally, in Section V, the conclusions of the paper and the future work are given.

\section{RELATED WORK}

This section gives an overview of the related work and the state-ofthe-art. This related work does not pretend to be exhaustive and for more details, a general overview of methods and models for Electric Load Forecasting in the last decades can be found in [ 3, $25,26]$. In contrast, this review focuses mostly on our research issues.

Hammad et al. [3] have reviewed over 15 different forecasting models distributed into 45 most relevant scientific papers about the electric load forecasting methods. Some of them are more appropriate and preferred such as regression analysis based models and artificial neural networks (ANN), which are the most utilized models in electricity predictions. In this scope, the artificial neural networks (ANN) models are mainly employed for short-term predictions where electricity and power consumption patterns are more complicated.

Antonopoulos et al. [25] have investigated over 160 papers, published between 2009 and 2019, as well as 40 companies and commercial initiatives, and 21 large projects to identify and discuss the trends for AI approaches in the energy Demand Response (DR) sector. The literature reviewed in this work display that AI approaches are a promising technology for DR applications. Going forward, adoption of AI is paramount for the wide success of DR schemes. Even though AI approaches offer tools to tackle many challenges of the DR schemes, they also pose a series of considerations and limitations. Better understanding of the methods and their limitations is vital for the proper application in the DR setting. This review highlighted that a large number of different AI techniques are being used, but it appears clearly that some techniques are more suitable than others for specific tasks. Indeed, it is showed that ANNs, which are commonly used for multi-variable function approximation and regression, are extensively used for short term load and price forecasting, using supervised learning to achieve accurate prediction. 
Chen et al. [26] have proposed theory-guided deep-learning load forecasting (TgDLF), which is a gradient-free model that fully combines domain knowledge and machine learning algorithms. TgDLF predicts the future load through load ratio decomposition, in which dimensionless trends are obtained based on domain knowledge, and the local fluctuations are estimated via data-driven models. TgDLF simplifies the problem with the assistance of expertise, and utilizes the strong expressive power of neural networks to obtain accurate predictions. The historical load, weather forecast and calendar effect are considered in the model, and the model's robustness to inaccurate weather forecast data is improved by adding synthetic disturbance during the training process. Cross-validation experiments demonstrate that TgDLF is $23 \%$ more accurate than long short-term memory, and the TgDLF with enhanced robustness can effectively extract information from weather forecast data with up to $40 \%$ noise.

According to Chen et al. [26], for time series data, the long shortterm memory (LSTM) performs better since it has a loop in its architecture, and it is able to preserve long-term previous information for future use. Load forecasting is a typical time series data problem, and the LSTM is the desired model. Therefore, many recent studies in the field of load forecasting are based on the LSTM model. Many researchers have utilized LSTM to forecast electricity demand and achieved good results [27, 28]. Sun et al. proposed a method to select the effective features in a LSTM model for load consumption forecasting in 2020 [29]. Nespoli et al. inspected the impact of data quality of different features on load forecasting accuracy of LSTM [30]. Hossain and Mahmood utilized LSTM to predict a single step ahead load and multi-step intraday rolling horizons based on weather data and calendar effect, and achieved better performance than other machine learning models in 2020 [31].

The LSTM has been proven to constitute the state-of-the-art model, and it is taken as one of the baselines in this article.

Modeling and simulating in the Smart Grid are not completely new topics to discuss about. Several publications deal with ModelDriven Development (MDD) in this domain [32, 33, 34], whereas others propose the possibility of simulating such a system $[35,36$, 37]. Binder et al. [38] have proposed an interface developed for the Smart Grid Architecture Model (SGAM) Toolbox in order to exchange the model data with the simulation framework Mosaik2 [39]. SGAM introduced by the European Standardization Mandate $\mathrm{M} / 490$, is an architecture model specification that gives a holistic view on Smart Grid systems. The SGAM provides capabilities for the comprehensible development of renewable energy systems. Figure 4 depicts an overview of the model. Based on the NIST Domain Model, the automation pyramid and the GWAC Interoperability Stack, the concepts have been extended to a threedimensional structure, including domain-specific viewpoints.

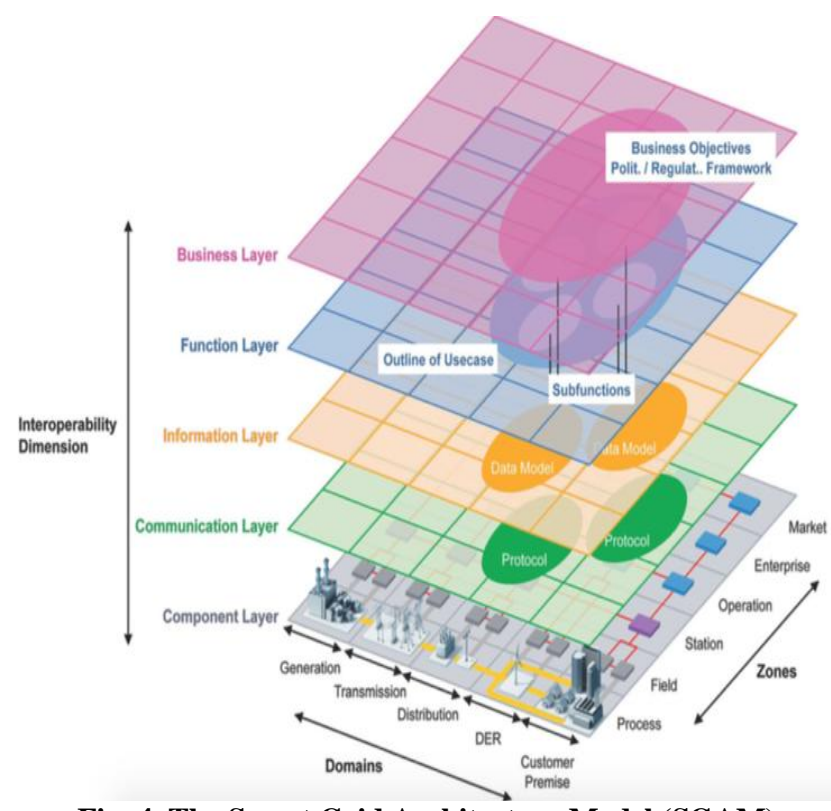

Fig. 4. The Smart Grid Architecture Model (SGAM)

The HOMER Pro® microgrid software by HOMER Energy [40] is the global standard for optimizing microgrid design in all sectors. Originally developed at the National Renewable Energy Laboratory, and enhanced and distributed by HOMER Energy, HOMER (Hybrid Optimization Model for Multiple Energy Resources) nests three powerful tools in one software product, so that engineering and economics work side by side.

HOMER is a simulation model. It will attempt to simulate a viable system for all possible combinations of the equipment that you wish to consider. Depending on how you set up your problem, HOMER may simulate hundreds or even thousands of systems.

- HOMER simulates the operation of a hybrid microgrid for an entire year, in time steps from one minute to one hour.

- Depending on the choice of forecasting method/ strategy and the parameter configuration the forecasting accuracy can be subject to significant variations.

In recent years, a highly detailed load profile generator called LoadProfileGenerator (LPG) [41] for private households has been developed, which is available as Microsoft desktop open source application [loadprofilegenerator.de]. LPG is an Agent-Based Behavior Simulation for Generating Residential Load Profiles The basic idea of behavioral simulation is shown in the Figure 5. The modeling approach is based on the premise that people act according to their needs. If a software agent is programmed in such a way that he tries to satisfy his needs as well as possible and is then offered appropriate activities as options, realistic behavior patterns emerge. Load profiles can then be created from the behavior patterns.

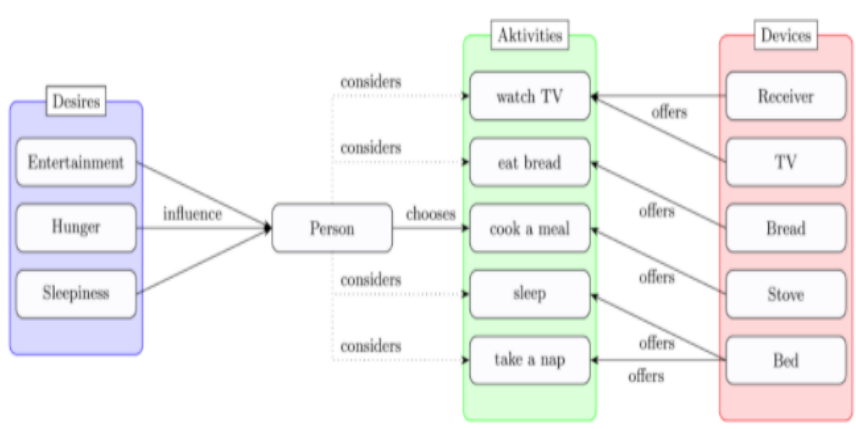

Fig. 5. The behaviour simulation load profile generator 
We conclude that deploying ILFM is seen to be complicated for both engineers and academician and is still an ongoing area of research. The key findings are summarised as follows:

- In the last decade, several studies (90\%) have applied Intelligent Load Forecasting Methods (ILFM) as compared with Conventional Load Forecasting Methods (CLFM), (10\%) to address energy prediction problems;

- However, not enough studies have been done in order to facilitate the deployment of Intelligent Load Forecasting Methods (ILFM).

\section{PROPOSED MODEL}

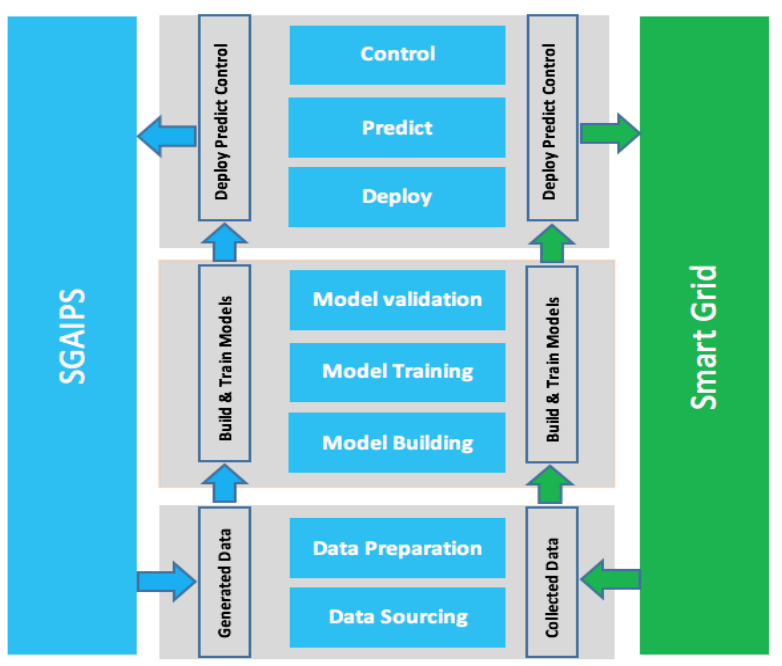

Fig. 6. The Smart Grid Artificial Intelligence Prediction Simulator Model.

As shown in Figure 6, we propose a Smart Grid Artificial Intelligence Prediction Simulator Model (SGAIPS-Model), in order to facilitate the deployment of Intelligent Load Forecasting Methods (ILFM).

SGAIPS-Model includes data sourcing data preparation, model building, model training, model validation. deployment, prediction and control. Data can be generated from simulation mode of SGAIPS or collected from Smart Grids via smart meters or others IoT devices.

The deployment of Intelligent Load Forecasting Methods (ILFM) involves three stages on which researchers and engineers collaborate:

1. Data acquisition and preparation

2. Build, train and validate the model

3. Deploy the model and predict electricity consumption, in order to control the system.

Data controlling can be used on two modes: Real mode (Smart Grid) or Simulation Mode (SGAIPS). It provides various benefits for managing the daily electric load demand and ensuring the supply / demand balance.

\section{DEPLOYMENT OF SGAIPS}

As mentioned in introduction, SGAIPS is a zero code SaaS Simulator for Smart Grid (Figure 7), based on TensorFlow, Keras and Flask, that aims to simplify the deployment of Intelligent Load Forecasting Methods (ILFM) on three main stages.

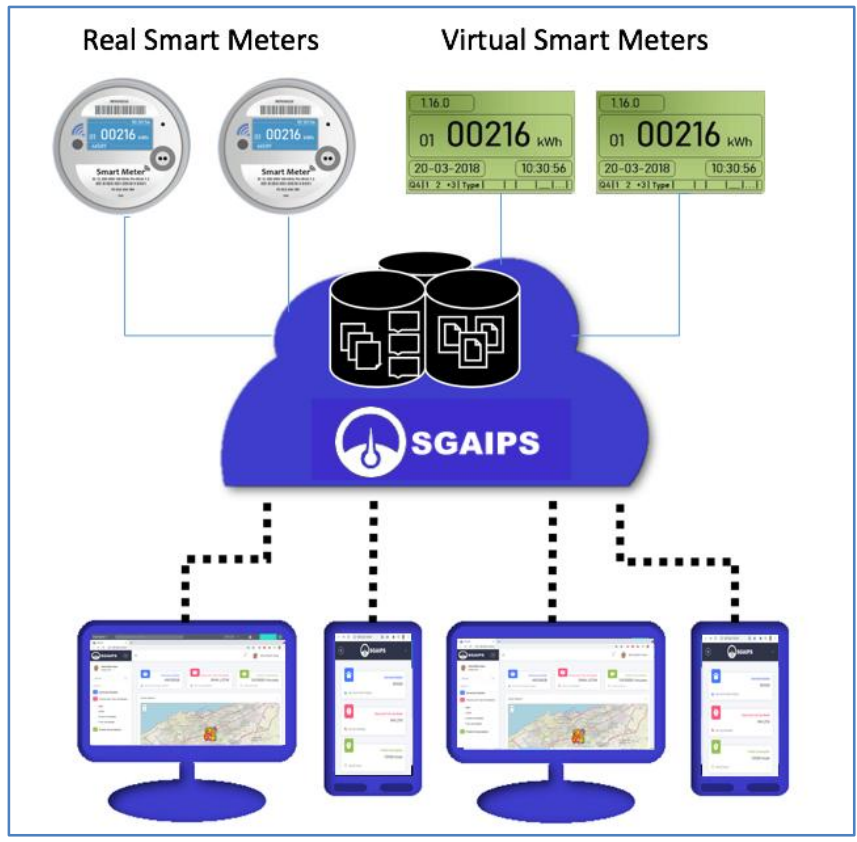

Fig. 7. Deployment of SGAIPS

\subsection{Data acquisition and preparation}

Data acquisition can be used on two modes: Real mode and Simulation Mode.

- In the real mode, smart meters play the main role on the customer side and are considered the first basis for the efficient operation of Smart Grids. On the provider side, data collected from smart meters can be used by SGAIPS.

- In simulation mode, as shown on Figure 8, researchers and engineers can generate virtual smart meters and their datasets in csv format or download them from the public datasets platform Kaggle [42].

Data preparation begins with the cleaning of data and ends with the data normalization.

In our case, we have used the simulation mode of SGAIPS. The dataset used in this work is a Kaggle dataset [43] with a data measurement frequency per hour and hourly energy consumption Over 10 years of megawatt power consumption data, the measurement period from 2004-10-01 to 2018-08-03. The total number of raw data points is 121271 , we can see in the table 1 below a visualization of the dataset (the dataset contains a set of regions, we choose the data collected from the Dayton Power and Light Company (DPLC)).

The time-series energy consumption data consist of two attributes such as date time and consumption. The date time attribute indicates the time at which the electrical energy is consumed. 
Table 1. Dataset Table

\begin{tabular}{|c|c|c|}
\hline & Datetime & DAYTON MW \\
\hline 0 & $2005-12-3101: 00: 00$ & 9389.0 \\
\hline 1 & $2005-12-3102: 00: 00$ & 9070.0 \\
\hline 2 & $2005-12-3103: 00: 00$ & 9001.0 \\
\hline 3 & $2005-12-3104: 00: 00$ & 9042.0 \\
\hline 4 & $2005-12-3105: 00: 00$ & 9132.0 \\
\hline$\ldots$ & $\ldots$ & $\ldots$ \\
\hline 121268 & $2018-08-0222: 00: 00$ & 2405.0 \\
\hline 121269 & $2018-08-0223: 00: 00$ & 2250.0 \\
\hline 121270 & $2018-08-0200: 00: 00$ & 2024.0 \\
\hline
\end{tabular}

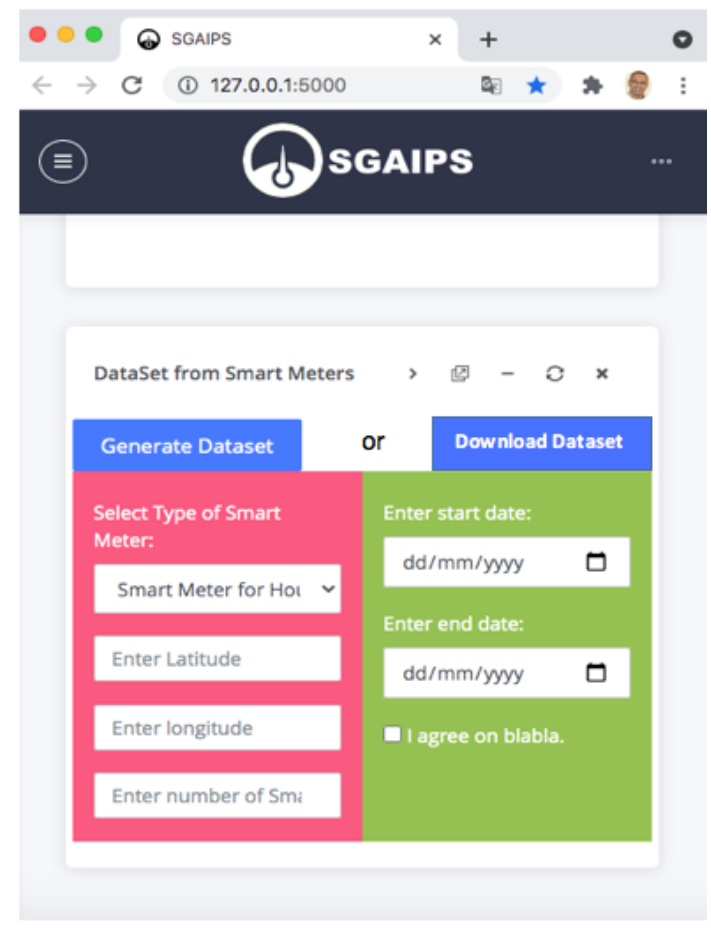

Fig. 8. The SGAIPS simulation mode.

\subsection{Build, train and validate the model}

Load forecasting is a typical time series data problem. As already mentioned in the literature review, for time series data, Recurrent Neural Network (RNN) and Long Short-Term Memory (LSTM) achieve better overall prediction results. Though enormous number of approaches have been proposed to improve the prediction results, we briefly describe the RNN, LSTM method, recurrent neural networks of which LSTM are

the most powerful and well-known subset, are a type of artificial neural network designed to recognize patterns in sequences of data, such as numerical times series data from sensors. What differentiates RNNs (Figure 9) and LSTMs (Figure 10) from other neural networks is that they take time and sequence into account, they have a temporal dimension.
Recurrent Neural Network: is basically a generalization of feedforward neural network that has an internal memory. RNNs are a special kind of neural networks that are designed to effectively deal with sequential data. This kind of data includes time series (a list of values of some parameters over a certain period of time) text documents, which can be seen as a sequence of words, or audio, which can be seen as a sequence of sound frequencies over time.

RNN is recurrent in nature as it performs the same function for every input of data while the output of the current input depends on the past one computation. For deciding, it considers the current input and the output that it has learned from the previous input [18].

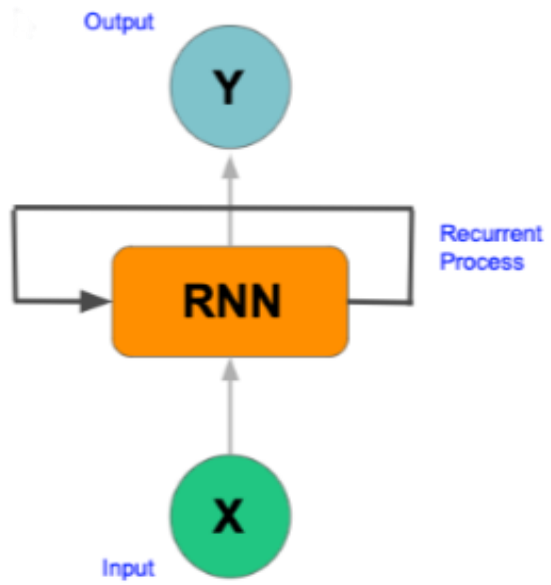

Fig. 9. The network architecture of RNN.

Long Short-Term Memory: LSTM is an improved version of the regular RNN which was designed to make it easy to capture longterm dependencies in sequence data. A regular RNN functions in such a way that the hidden state activation is influenced by the other local activations nearest to them, which corresponds to a Short-Term Memory, while the network weights are influenced by the computations that take place over entire long sequences, which corresponds to Long-Term Memory. Hence the RNN was redesigned so that it has an activation state that can also act as weights and preserve information over long distances, hence the name Long Short- Term Memory [19].

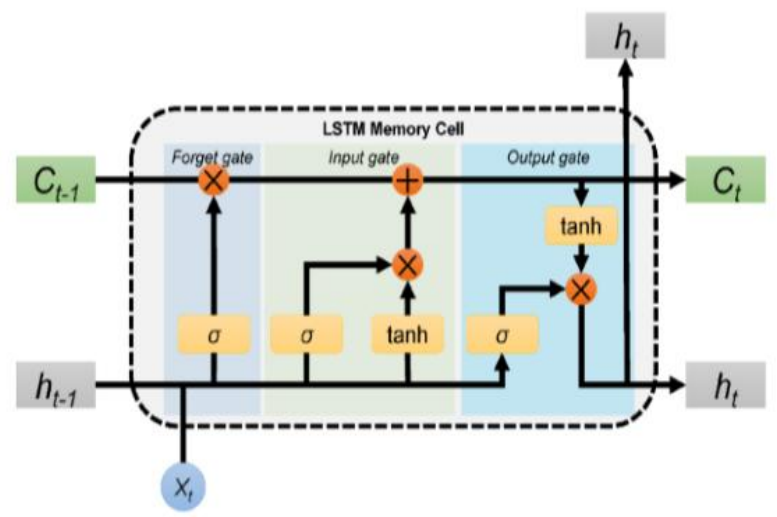

Fig. 10. The network architecture of LSTM.

As shown in Figure 11, we can easily build, train and validate a RNN and LSTM model with a simple Web app. We must simply specify the next parameters:

- $\quad$ Number of RNN or LSTM Layers

- Number of block on each layer 
- $\quad$ Sequence length

- Activation Function

- Optimizer

- Number of epochs

- Batch size

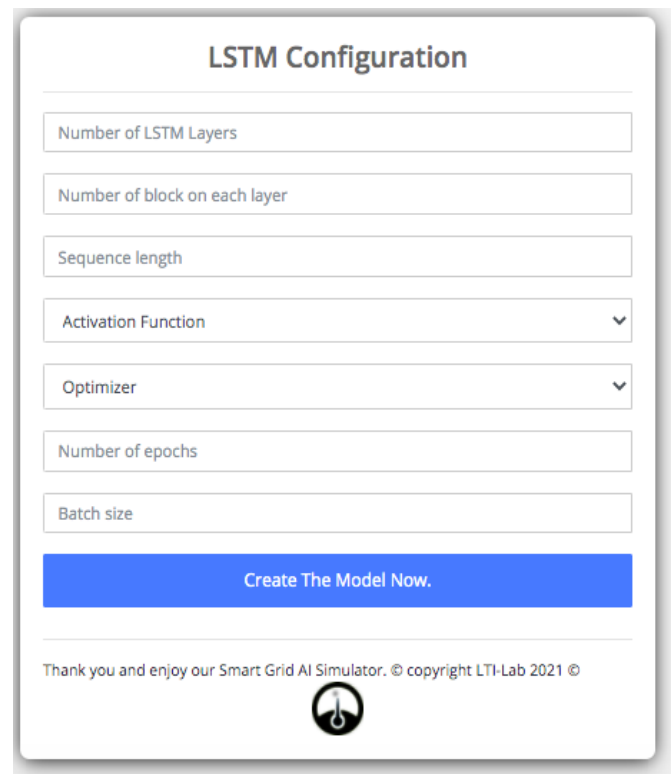

Fig. 11. The LSTM build form

In our case, we have build a RNN and LSTM models with 3 layers, each layer is composed of 40 blocks, 20 sequence length, we have used tanh activation function for each hidden layer and the third layer is followed by a dense layer. We have used Adam optimizer with Mean Squared Error (MSE) loss function, and fit the model for 10 epochs with a batch size of 1000. After training the model, we saved it as a Keras H5 format.

\section{3 . Deploy, Predict and Control}

Deploying machine learning models in production is not a discussed enough issue. SGAIPS is designed to facilitate the deployment of trained model and make it available to the end users on the front end side. On the backend side, SGAIPS is based on Flask that is a Python Web framework built for rapid development.

In the prediction stage, we compare the actual values vs predicted values by plotting a graph. We use also $\mathrm{R}^{2}$ regression score function, the coefficient of determination, noted $\mathrm{R}^{2}$ or $\mathrm{r}^{2}$, is a measure of the quality of the prediction of a linear regression. It is defined by:

$$
R^{2}=1-\frac{\sum_{i=1}^{n}\left(y_{i}-\hat{y}_{i}\right)^{2}}{\sum_{i=1}^{n}\left(y_{i}-\bar{y}\right)^{2}}
$$

Where $\mathrm{n}=$ number of measurements

$$
\begin{aligned}
& y_{i}=\text { value of measure }{ }^{\circ} i \\
& \hat{y}=\text { corresponding predicted value } \\
& \bar{y}=\text { average of the measurements }
\end{aligned}
$$

In our case, we see in Figure 12 that the predicted values are close to the actual values, which means that the RNN model works well to predict the sequence. $\mathrm{R}^{2}$ score for values predicted by the trained RNN model equal to 0.9541 . Noted that the best possible score is 1.0 .

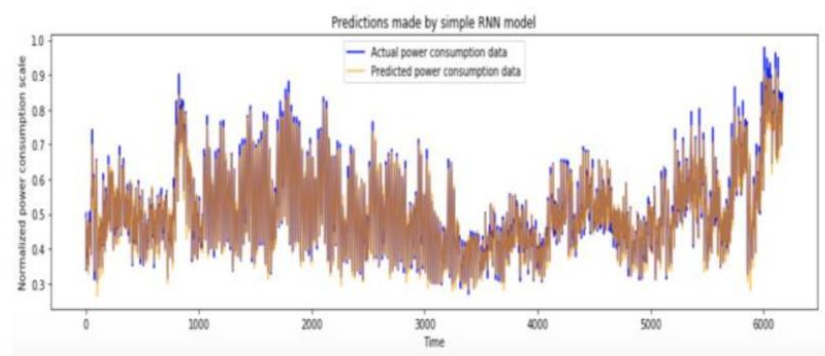

Fig. 12. Prediction by RNN

Using the LSTM method, as shown on Figure 13, the prediction function is closer to the real value, $\mathrm{R}^{2}$ score for the values predicted by the trained LSTM model equal to 0.9553 .

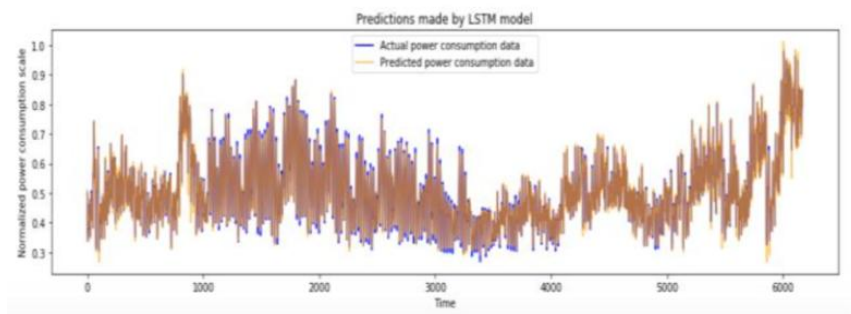

Fig.13. Prediction by LSTM

For better clarification we compare predictions made by simple RNN, LSTM model by plotting data in a single graph as shown below in the Figure 14:

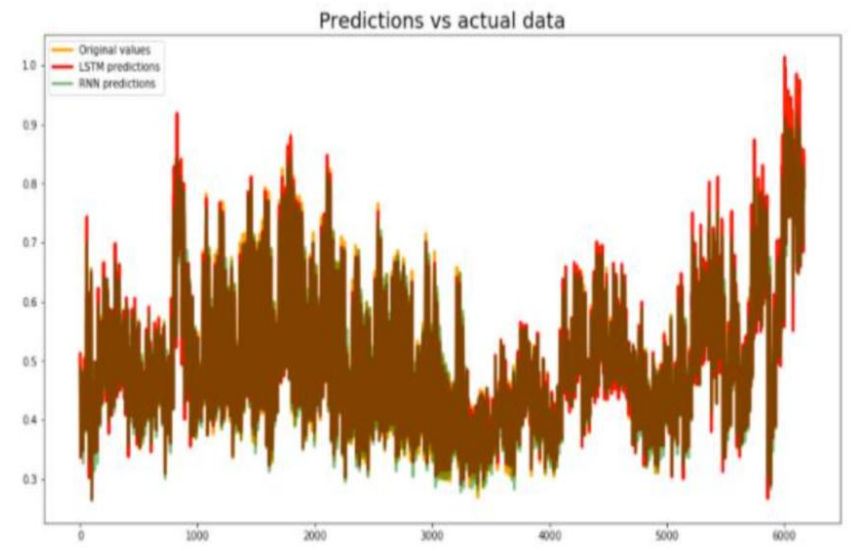

Fig. 14. Predictions comparison

Accurate electricity load forecasting is an essential part of control in smart grid in order to retain high energy efficiency and reliable operation. Of course, there is always a chance of unforeseen load jumps, yet more important is the application of intelligent load forecasting deployed model that could minimize the number of such incidents by careful analysis of historical trends.

\section{CONCLUSION}

In this article, we have built a new Smart Grid Artificial Intelligence Prediction Simulator called SGAIPS, delivered as SaaS (software-as-a-Service), that aims to simplify the deployment of Intelligent Load Forecasting Methods (ILFM) in smart grids. We focused on the problem of short-term load forecasting using ILMF and their deployment.

After giving an overview of the related work and the state-of-theart. We have concluded that deploying ILFM is seen to be complicated for both engineers and academician and is still an ongoing area of research. 
Before deploying ILMF, we have proposed a new model called SGAIPS-Model that includes data sourcing data preparation, model building, model training, model validation, model deployment, prediction and control stages.

In the end, we have shown that it is extremely easy to use SGAIPS for short term electrical load prediction based on RNN and LSTM deep learning models.

In future work, we will extend the experiment of SGAIPS and we will see if we can improve the prediction by adding other input parameters like weather and other useful deep learning models.

\section{REFERENCES}

[1] F. Mountassir, R. Mali, A. Lmouatassime, M. Bousmah, "Machine Learning and IoT for Smart Grid" In Proceedings of the 5th International Conference on SMart City Applications (SCA), Safranbolu, Turkey, 7-9 October 2020.

[2] Lemuel Clark P. Velasco, Daisy Lou L. Polestico, Gary Paolo O. Macasieb, Michael Bryan V. Reyes and Felicisimo B. Vasquez Jr , "Load Forecasting using Autoregressive Integrated Moving Average and Artificial Neural Network" International Journal of Advanced Computer Science and Applications(ijacsa), 9(7), 2018. http://dx.doi.org/10.14569/IJACSA.2018.090704

[3] A. Hammad, B. Jereb, B. Rosi, D. Dragan., "Methods and Models for Electric Load Forecasting: A Comprehensive Review," Logistics \& Sustainable Transport, vol.11, no.1, 2020, pp.51-76. https://doi.org/10.2478/jlst-2020-0004

[4] E. Almeshaiei and H. Soltan, "A Methodology for Electric Power Load Forecasting," Alexandria Engineering Journal, vol. 50, no. 2011, pp. 137-144, 2011.

[5] J. Zhang, "Research on Power Load Forecasting Based on the Improved Elman Neural Network," The Italian Association of Chemical Engineering (AIDIC), vol. 51, no. 2016, pp. 589594, 2016.

[6] Ü. B. Filik, Ö. N. Gerek, and M. Kurban, "A Novel Modeling Approach for Hourly Forecasting of Long-Term Electric Energy Demand," Energy Conversion and Management, vol. 52, no. 2011, pp. 199-211, 2011.

[7] R. Gordillo-Orquera, L. M. Lopez-Ramos, S. Muñoz-Romero, P. Iglesias-Casarrubios, D. Arcos-Avilés, A. G. Marques, and J. L. Rojo-Álvarez, "Analyzing and Forecasting Electrical Load Consumption in Healthcare Buildings," Energies, vol. 11, no. 493, 2018.

[8] G. Nalcaci, A. Özmen, and G. W. Weber, "Long-term Load Forecasting: Models Based on MARS, ANN and LR methods," Central European Journal of Operations Research (CEJOR), Springer-Verlag GmbH Germany, vol. 27, no. 2019, pp. 1033-1049, 2018.

[9] L. Friedrich and A. Afshari, "Short-Term Forecasting of the Abu Dhabi Electricity Load Using Multiple Weather Variables," presented at the 7th International Conference on Applied Energy (ICAE), 2015.

[10] R. Wanga, J. Wangb, and Y. Xu, "A Novel Combined Model Based on Bybrid Optimization Algorithm for Electrical Load Forecasting," Applied Soft Computing Journal, vol. 82, no. 2019, p. 105548, 2019.

[11] N. Abu-Shikhah and F. Elkarmi, "Medium-Term Electric Load Forecasting Using Singular Value Decomposition," Energy Conversion and Management, vol. 36, no. 7, pp. 4259-4271, 2011.
[12] Lei Zhang, Linghui Yang, Chengyu Gu, Da Li, "LSTMbased Short-term Electrical Load Forecasting and Anomaly Correction," E3S Web of Conferences182,01004(2020), https://doi.org/10.1051/e3sconf/202018201004

[13] G. E. P. Box, G. M. Jenkins, and G. C. Reinsel, " Time series analysis: Forecasting and control ," 4th ed. ed. (Wiley series in probability and statistics). Oxford: Wiley, 2008.

[14] P. S. P. Cowpertwait and A. V. Metcalfe, "Introductory Time Series with R". Springer New York, 2009.

[15] A. J. Wood, B. F. Wollenberg, and G. B. Sheblé, "Power Generation, Operation, and Control." Wiley, 2013.

[16] Fu Y, Li Z, Zhang H, et al. "Using support vector machine to predict next day electricity load of public buildings with submetering devices." Procedia Engineering 121: 1016-1022 (2015).

[17] J. G. Jetcheva, M. Majidpour, W. P. Chen, "Neural network model ensembles for building-level electricity load forecasts," Energy and Buildings 84, 214-223 (2014).

[18] J. Ku, R. Goomer, A. K. Singh, "Long Short Term Memory Recurrent Neural Network (LSTM-RNN) Based Workload Forecasting Model For Cloud Datacenters," Procedia Computer Science 125, 676- 682 (2018).

[19] Y. H. Zhang, C. M. Qiu, X. He, et al., "A Short-Term Load Forecasting Based on LSTM Neural Network," Electric Power Information \& Communication Technology, (2017).

[20] Delivery, Kee Yuan Ngiam, Ing Wei Khor, "Big data and machine learning algorithms for health-care," Lancet Oncol 2019; 20: e262-73.

[21] Jafar Alzubi, Nayyar Anand, Kumar Akshi, "Machine Learning from Theory to Algorithms: An Overview," $2018 \mathrm{~J}$. Phys.: Conf. Ser. 1142012012.

[22] Bengio Y., Courville A., Vincent P.,"Representation Learning: A Review and New Perspectives,". IEEE Transactions on Pattern Analysis and Machine Intelligence (2013). $35 \quad$ (8): $1798-1828 . \quad$ arXiv:1206.5538. doi:10.1109/tpami.2013.50. PMID 23787338. S2CID 393948.

[23] Schmidhuber J., "Deep Learning in Neural Networks: An Overview". Neural Networks. 61: 85-117(2015). arXiv:1404.7828. doi:10.1016/j.neunet.2014.09.003. PMID 25462637. S2CID 11715509.

[24] Bengio Yoshua, LeCun, Yann, Hinton Geoffrey (2015). "Deep Learning". Nature. 521 (7553): 436-444. Bibcode:2015 Natur.521..436L. doi:10.1038/nature14539. PMID 26017442. S2CID 3074096.

[25] Antonopoulos, I, Robu, V, Couraud, B, Kirli, D, Norbu, S, Kiprakis, A, Flynn, D, Elizondo-González, S \& Wattam, S 2020, "Artificial intelligence and machine learning approaches to energy demand-side response: A systematic review", Renewable and Sustainable Energy Reviews, vol. 130, 109899. https://doi.org/10.1016/j.rser.2020.109899

[26] Yuntian Chen, Dongxiao Zhang, "Theory-guided deeplearning for electrical load forecasting (TgDLF) via ensemble long short-term memory", Advances in Applied Energy, Volume 1, 2021, 100004.

[27] Bedi J, Toshniwal D. "Deep learning framework to forecast electricity demand”, Appl Energy 2019;238:1312-26.

[28] Kwon BS, Park RJ, Song KB. "Short-term load forecasting based on deep neural networks using LSTM layer", J Electr 
Eng Technol 2020;15:1501-9. doi:10.1007/s42835-02000424-7.

[29] Sun G, Jiang C, Wang X, Yang X, "Short-term building load forecast based on a data- mining feature selection and LSTMRNN method", IEEE Trans Electr Electron Eng 2020;15(7):1002-10. doi:10.1002/tee.23144.

[30] Nespoli A, Ogliari E, Pretto S, Gavazzeni M, Vigani S, Paccanelli F, "Data quality analysis in day-ahead load forecast by means of LSTM", Proceedings of the IEEE international conference on environment and electrical engineering and IEEE industrial and commercial power systems Europe (EEEIC/I\&CPS Europe). IEEE; 2020.

[31] Hossain MS, Mahmood H, "Short-term load forecasting using an LSTM neural network", Proceedings of the IEEE power and energy conference at Illinois (PECI). IEEE; 2020.

[32] Lampropoulos I, Vanalme GM, Kling WL, "A methodology for modeling the behavior of electricity prosumers within the smart grid", 2010 IEEE PES Innovative Smart Grid Technologies Conference Europe (ISGT Europe). IEEE, Gothenberg, pp 1-8

[33] Lopes AJ, Lezama R, Pineda R, "Model based systems engineering for smart grids as systems of systems", Procedia Comput Sci 6:441-450, 2011.

[34] Andrén F, Stifter M, Strasser T, "Towards a semantic driven framework for smart grid applications: Model-driven development using cim", iec 61850 and iec 61499 . Informatik-Spektrum 36(1):58-68, 2013.

[35] Godfrey T, Mullen S, Griffith DW, Golmie N, Dugan RC, Rodine C "Modeling smart grid applications with cosimulation",First IEEE International Conference on Smart
Grid Communications. IEEE, Gaithersburg, pp 291-296, $\{2010)$.

[36] Yang C-H, Zhabelova G, Yang C-W, Vyatkin V, "Cosimulation environment for event-driven distributed controls of smart grid", IEEE Trans Ind Inf 9(3):1423-1435, (2013).

[37] Palensky P, Widl E, Elsheikh A, "Simulating cyber-physical energy systems: Challenges, tools and methods", IEEE TransSyst Man Cybern Syst 44(3):318-326, (2013).

[38] Christoph Binder, Michael Fischinger, Lukas Altenhuber, Dieter Draxler, Goran Lastro and Christian Neureiter, "Enabling architecture based Co-Simulation of complex Smart Grid applications", Energy Informatics (2019), 2 (Suppl1): 20, https://doi.org/s42162-019-0084-0

[39] Schütte S, Scherfke S, Tröschel M, "Mosaik: A framework for modular simulation of active components in smart grids", Smart Grid Modeling and Simulation (SGMS), 2011 IEEE First International Workshop On. IEEE, Brussels. pp 55-60.

[40] The HOMER $\quad$ Pro ${ }^{\circledR}$ microgrid. https://www.homerenergy.com/products/pro/index.html

[41] The Load Profile Generator. https://www.loadprofilegenerator.de/

[42] Kaggle: The world's largest data science community https://www.kaggle.com/

[43] Rob Mulla, "Over 10 years of hourly energy consumption data from PJM in Megawatts", Kaggle, 2018. https://www.kaggle.com/robikscube/hourly-energyconsumption 